УДК 536.7

Л. И. Морозюк

Одесская национальная академия пищевых технологий, ул. Канатная, 112,г. Одесса,65039

\title{
ТЕРМОДИНАМИЧЕСКИЙ АНАЛИЗ КАСКАДНЫХ ХОЛОДИЛЬНЫХ МАШИН С R744 В ВЕРХНЕМ КАСКАДЕ
}

\begin{abstract}
Каскадная холодильная машина - комплекс одноступенчатых ичиклов-каскадов с разными рабочими веществами. Приведен способ термодинамического анализа каскадных машин, в которых цикл верхнего каскада реализуется в надкритической области рабочим веществом R744,nри изменении температуры в конденсаторе-испарителе и давления в газовом охладителе, Установлено влияние указанных параметров на объемные и энергетические характеристики сложного ицикла и машины в целом.
\end{abstract}

Ключевые слова: каскадная холодильная машина-R744-надкритический ичил- объемные и энергетические характеристики машинь

\section{Л. І. Морозюк}

Одеська національна академія харчових технологій, вул. Канатна, 112, м. Одеса, 65039

\section{ТЕРМОДИНАМІЧНИЙ АНАЛІЗ КАСКАДНИХ ХОЛОДИЛЬНИХ МАШИН 3 R744 У ВЕРХНЬОМУ КАСКАДІ}

Каскадна холодильна машина - комплекс з одноступеневих ичклів-каскадів з різними робочими речовинами. Наведено спосіб термодинамічного аналізу каскадних машин, в яких ичик верхнього каскаду здійснюється в надкритичній області робочою речовиною $R 744$, за зміною температури в конденсаторі-випарнику та тиску в газовому охолоджувачі. Встановлено вплив вказаних параметрів на об 'ємні та енергетичні характеристики складного цчиклу та машини в цілому

Ключові слова: каскадна холодильна машина - R744 - надкритичний цикл-об'ємні та енергетичні характеристики машини

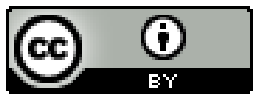

This work is licensed under the Creative Commons Attribution International License (CC BY). http://creativecommons.org/licenses/by/4.0/

\section{І. ВВЕДЕНИЕ}

Низким температурам кипения и высоким температурам конденсации рабочего вещества в холодильных машинах соответствуют малые величины удельной объемной холодопроизводительности, низкие значения объемных и энергетических коэффициентов компрессора, которые непосредственно влияют на холодопроизводительность $Q_{0}$ и потребляемую мощность $N_{e}$. В результате при определенном температурном режиме получение холода в простой одноступенчатой машине становится невозможным. Распределение температурных режимов и схемных решений с учетом объемных, массогабаритных и энергетических характеристик компрессоров, комплектующих машины, осуществляется между многоступенчатыми машинами с одним рабочим веществом и каскадными, работающими с двумя и более рабочими веществами. При этом массовые расходы рабочих веществ по ступеням или каскадам различные, что и определяет сложное схемно-цикло-вое решение холодильной машины.

Рабочими веществами в двухступенчатых машинах служили $R 22$ и $R 717$, в каскадных - R12 и $R 22$ в высокотемпературном верхнем каскаде $(B K)$ и $R 13$, $R 14$ - в низкотемпературном нижнем каскаде (HK). $\mathrm{C}$ точки зрения потребительских характеристик машины (холодопроизводительности $Q_{0}$ и потребляемой мощности $N_{e}$ ) чистые вещества и пары веществ удовлетворяли требованиям энергосбережения в холодильной технике.

Однако в современной холодильной технике, указанные рабочие вещества запрещены к использованию. Это привело к ограничению реализуемых температур кипения в циклах двухступенчатого сжатия до $-50{ }^{\circ} \mathrm{C}$, связанного со свойствами $R 717$, и поиску новых пар рабочих веществ для каскадных машин, отвечающих совместным требованиям энергосбережения и экологической без-опасности, для обеспечения температур в объектах от $-110{ }^{\circ} \mathrm{C}$ до $-50{ }^{\circ} \mathrm{C}$.

Научно-техническая информация о поиске новых рабочих веществ и создании нового поколения схемно-цикловых решений каскадных холодильных машин для них содержится в работах последнего десятилетия.

Наиболее известной и изученной является машина с парой веществ $R 717 / R 744$ (аммиак $R 717$ - 
в $B K$, диоксид углерода $R 744$ - в $H K)$. Термодинамический анализ сложного цикла машины установил, что перспективной областью использования являются машины крупной холодопроизводительности при температурах в охлаждаемых объектах от $-50{ }^{\circ} \mathrm{C}$ до $30^{\circ} \mathrm{C}$.

Рассматривается и возможная замена $R 717$ на $R 290$ (пропан), $R 1270$ (пропилен) и $R 404 A$, при этом использование $R 744$ в качестве рабочего вещества $B K$ не предполагалось по причине высоких рабочих давлений при реализации цикла в надкритической области [1-3].

Машины выпускают в промышленном масштабе ведущие мировые фирмы [4].

Работа [5], в которой рассмотрена каскадная машина с веществами $R 744 / R 290$, предлагает $R 744$ в качестве рабочего вещества $B K$ с циклом в надкритической области. Авторы приводят характеристики сложного цикла при решении «энергетической задачи», т.е. определении максимального коэффициента преобразования COP машины как функции промежуточной (граничной) температуры $T_{\kappa и}$ между $B K$ и $H K$. При этом главными преимуществами пары отмечены следующее факторы: оба вещества натуральные, имеют нулевые значения ODP и близкие к нулю GWP, обеспечивают температурный уровень от $-60{ }^{\circ} \mathrm{C}$ до $-30{ }^{\circ} \mathrm{C}$.

В работе [6] представлен анализ нескольких пар веществ, в которых одним является $R 744$, а группу веществ $H F C$-типа предполагается использовать в HК для получения температур ниже температуры тройной точки $R 744\left(-56,6^{\circ} \mathrm{C}\right)$. В группу вошли $R 125$, $R 41, R 32$ и $R 23$. В этой же работе приведена информация об экспериментальных исследованиях каскадных машин с азеотропными смесями R170/R23 и $R 170 / R 116$ в $H K$ с температурами ниже $-80^{\circ} \mathrm{C}$. Смеси рассмотрены как альтернатива $R 508$.

C точки зрения схемно-циклового решения каскадной холодильной машины, интересной является работа [7]. В ней рассмотрено использование $R 744$ одновременно в обоих каскадах. $B K$ работает по надкритическому циклу, $H K$ - по циклу в области температур ниже тройной точки (trans - triplecycle). Дросселирование в $H K$ осуществляется в двухфазную зону «пар-твёрдое тело» с осаждением сухого льда внутри испарителя. При подводе тепла сухой лёд сублимирует, а каскадная машина работает непрерывно со стабильным температурным режимом в испарителениже- $56,6^{\circ} \mathrm{C}$.

Во всех работах рассмотрена только«энергетическая задача» с выводами, что при фиксированных крайних температурах каскадного цикла пары рабочих веществ не равноценны во влиянии на энергетические характеристики машин. Это влияние оценено абсолютной величиной коэффициента преобразования COP машины в зависимости от $T_{\kappa u}$. Такой подход к анализу машины необходим, но недостаточен. С точки зрения энергетической эффективности, влияние должно быть оценено относительной величиной - степенью термодинамического совершенства рабочего вещества $\eta_{\text {cmc }}$. Массогаба- ритные (инвестиционные) характеристики машины должны быть также оценены, но путем решения двух задач - «транспортной» и «унификации». Следует отметить, что с $R 744$ в верхнем каскаде коэффициент преобразования $C O P$ машины в целом будет зависеть и от давления в газовом охладителе $B K$, поэтому влияние должно быть оценено как:

$$
C O P=f\left(T_{\kappa и}, p_{\text {охл }}^{6 \kappa}\right) .
$$

В связи с этим автор ставит и решает задачу поиска схемно-цикловых решений для каскадных машин с надкритическим циклом $B K$, базирующуюся на общем методе сравнительной оценки термодинамических параметров, разработанном Р. Планком [8]. Метод - удобное средство для оценки преимуществ того или иного схемно-циклового решения в конкретных условиях. Он не единственный, но существенный фактор, определяющий практическую целесообразность реализации схемно-циклового решения машин различных типов и назначения, поиска и внедрения новых технических решений.

\section{II. ОСНОВНАЯ ЧАСТЬ}

\section{Принципы выбора промежуточной температу- ры $\boldsymbol{T}_{\text {ки }}$} ке 1.

Принципиальная схема представлена на рисун-

Выбор промежуточной температуры $T_{\kappa и}$ может осуществляться по минимизации эффективной мощности, затрачиваемой компрессорами дов $\sum N_{e}=\min$, что соответствует максимальному значению степени термодинамического совершенства цикла каскадной машины $\eta_{c m c}$. Такая задача оптимизации носит название «энергетической задачи» и применяется при проектировании крупных систем хладоснабжения, где стоимость холода включается в стоимость продукции.

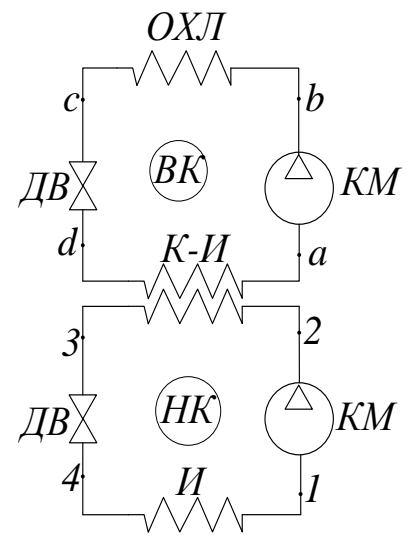

Рисунок 1 - Принципиальная схема каскадной машины: $K M$ - компрессор; $И$ - испаритель; $К И-$ конденсатор-испаритель; ДВ - дроссель; ОХЛ - газовый охладитель; $H K$ - нижний каскад; $B K$ - верхний каскад. 
Известно, что для любого транспорта служебное оборудование должно занимать минимально возможную площадь (объем), чтобы выделить максимальный объем для перевозимого груза. В связи с этим, задачу минимизации массогабаритных характеристик оборудования называют «транспортной задачей». В таком случае в каскадном цикле необходимо установить такое значение $T_{\kappa u}$, при котором минимизированы суммарные теоретические объемные производительности компрессоров $\sum V_{h}=\mathrm{min}$, a, следовательно, и мас-согабаритные характеристики всего остального оборудования.

«Задача унификации» решает проблему эксплуатационной надежности проектируемой холодильной машины. Подобные задачи решаются для специальных типов холодильных машин, например, применяемых в военно-промышленном комплексе.

В холодильных машинах наиболее уязвимым элементом является компрессор, поэтому задача унификации сводится к подбору одинаковых или унифицированных компрессоров. В этом случае при анализе каскадной машины устанавливается не только промежуточная температура $T_{\kappa u}$, но и создается схемно-цикловое ее решение. Задача важна для машин, в которых в обоих каскадах используют $R 744$.

\section{Построение алгоритма решения задачи}

Начальный этап решения задачи рассмотрен в работе [9], в которой автор представила способ определения степени термодинамического совершенства $\eta_{\text {стс }}$ сложного действительного цикла каскадной машины. На примере трех рабочих веществ $R 744, R 717, R 290$, при различных сочетаниях их в циклах-каскадах, выполнено сравнение термодинамического совершенства пар, показано взаимное влияние свойств рабочих веществ в каскадах на совершенство машины в целом. Термодинамический анализ выполнен при условии реализации цикловкаскадов в двухфазных областях

Новый этап в решении задачи - реализация цикла $B K$ в надкритической области (рисунок 2).

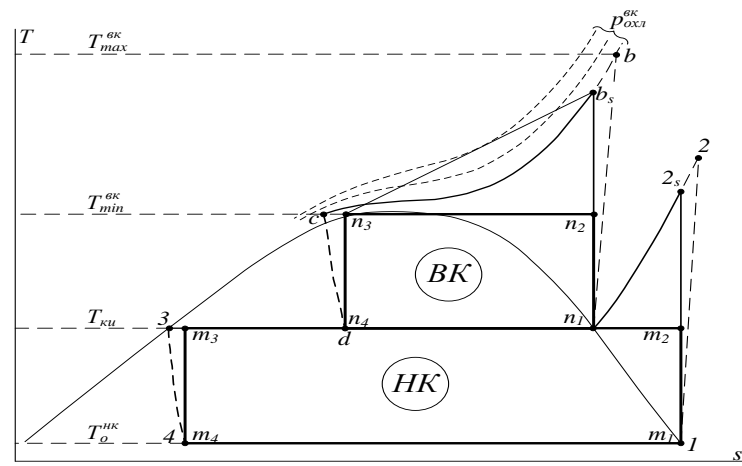

Рисунок 2 - Цикл машины с единым веществом в каскадах при $\Delta T_{\text {ки }}=0: T_{\max }^{\mathrm{B \kappa}}, T_{\min }^{\mathrm{B \kappa}}-$ максимальная и минимальная температуры в газовом охладителе; $T_{0}^{\text {нк }}$ - температура кипения в $H K ; T_{\kappa и}-$ температура в конденсаторе-испарителе.
Действительный цикл каскадной машины формируется из двух циклов Планка: цикл 1-2-3-4 НК и изикл $a-b-c-d B K$. В этом случае состояния рабочих веществ на всасывании в компрессоры и перед дросселированием однозначно определены. При наличии одного и того же рабочего вещества в обоих каскадах и отсутствии разности температур в конденсаторе-испарителе цикл является простейшим.

В такой машине циклы каскадов осуществляются в интервалах $\left[\left(T_{\max }^{\mathrm{B \kappa}} \ldots T_{\min }^{\mathrm{B \kappa}}\right) \ldots T_{\text {ки }}\right]$ в $B K \quad$ и $\left(T_{\text {ки }} \ldots T_{0}^{\text {нк }}\right)$ в $H K$ с разными массовыми расходами рабочих веществ. Уровень рабочих давлений в $H K$ соответствует обычным одноступенчатым циклам. Температуры и давления в $B K$ превышают критические значения, поэтому соответственный обратимый цикл для каскадной машины должен учитывать все особенности цикла с $R 744$ и сохранять тот же полезный эффект, что и в действительном цикле.

В цикле ВК процесс подвода тепла осуществляется при постоянной температуре (nроцесс $d$-a), процесс отвода тепла (процесс 4-1) при переменной температуре, в связи с этим в цикле-образце ход температур должен копировать рабочее вещество. В таких случаях циклом-образцом может служить сочетание циклов: «треугольный» цикл Лоренца $n_{2} b_{2} n_{3}$ и соответственный цикл Карно $n_{1} n_{2} n_{3} n_{4}$. Процесс отвода тепла в цикле Лоренца должен иметь постоянную теплоемкость [10]. Для R744 в области критической точки существует аномалия, наблюдается фазовый переход второго рода со значительным изменением изобарной теплоемкости [11], поэтому корректно было бы цикл $n_{2} b_{2} n_{3}$ разделить на несколько циклов, в которых теплоемкость можно считать не зависящей от температуры. На данном этапе анализа упростим задачу, и в качестве обратимого цикла-образца оставим только соответственный цикл но $n_{1} n_{2} n_{3} n_{4}$.

В диаграмме состояний $T-s$ (рисунок 2) действительный цикл представлен двумя контурами 1-23-4 и $a-b-c-d$, а соответственные циклы Карно $B K$ и $H K$ контурами и $n_{1}-n_{2}-n_{3}-n_{4}$ и $m_{1}-m_{2}-m_{3}-m_{4}$.

Для конкретных рабочих веществ и температурных режимов удельные характеристики цикла определяют с применением методики, представленной в работе [9]. В результате:

- массовый расход рабочего вещества $a$ верхнего каскада, отнесённый к 1 к рабочего вещества нижнего (в соответствии с энергетическим балансом кон-

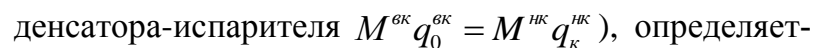
ся как:

$$
a=q_{\kappa}^{н \kappa} / q_{0}^{B \kappa}
$$

- коэффициент преобразования $C O P_{\partial}$ действительного цикла:

$$
C O P_{\partial}=q_{0}^{\eta \kappa}\left(w_{\kappa}^{\eta \kappa}+a w_{\kappa}^{\varepsilon \kappa}\right)^{-1},
$$

где $w_{\kappa}$-удельная действительная работа сжатия компрессоров $H K$ и $B K$;

- коэффициент преобразования $C O P_{\kappa}$ каскадной машины, работающей по циклу Карно - Карно:

$$
C O P_{\kappa}=q_{0}^{\imath \kappa}\left(w_{u}^{\imath \kappa}+a w_{u}^{6 \kappa}\right)^{-1},
$$


где $w_{u}$ - работа цикла Карно $H K$ и $B K$ соответственно;

- степень термодинамического совершенства:

$$
\eta_{c m c}=C O P_{\partial} / C O P_{\kappa}=\left(w_{u}^{u \kappa}+a \cdot w_{u}^{B \kappa}\right)\left(w_{\kappa}^{H \kappa}+a w_{\kappa}^{B \kappa}\right)^{-1} .
$$

Отыскание $\eta_{c m c}$ для различных пар веществ и сопоставление этих значений при фиксированном полезном эффекте $Q_{0}^{\text {нк }}$ в заданном температурном режиме является решением «энергетической задачи».

Как известно, термодинамическое совершенство цикла для $R 744$ в надкритической области при фиксированной температуре кипения зависит от давления в охладителе [8], поэтому термодинамический анализ должен быть проведен для различного соче-

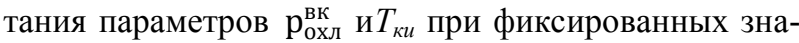
чениях $T_{\min }^{\mathrm{B \kappa}}$ и $T_{0}^{\mathrm{H \kappa}}$

«Транспортная задача» решается в тепловом расчете цикла каскадной машины. Конечной расчетной функцией является $\left(V_{h}^{u \kappa}+V_{h}^{\varepsilon \kappa}\right)=f\left(T_{\kappa и}\right)$. Отнесенная в 1 кг массового расхода рабочего вещества $H K$, суммарная объемная теоретическая производительность компрессоров определяется выражением:

$$
\sum V_{h} / M^{\mu \kappa}=\frac{v_{B c}^{H \kappa}}{\lambda^{H \kappa}}+\frac{a v_{B c}^{6 \kappa}}{\lambda^{6 \kappa}},
$$

где $v$ - удельный объем паров на всасывании в компрессор соответствующего каскада;

$\lambda$ - коэффициент подачи компрессора соответствующего каскада.

«Задача унификации» предполагает вычисление функции:

$$
\left(V_{h}^{H \kappa} / V_{h}^{B \kappa}\right)\left(M^{H \kappa}\right)^{-1}=\frac{1}{a} \frac{v_{B c}^{H \kappa}}{v_{B c}^{6 \kappa}} \frac{\lambda^{6 \kappa}}{\lambda^{H \kappa}}
$$

\section{III. ПРИМЕР РЕШЕНИЯ ЗАДАЧИ}

В качестве примера решим «энергетическую» задачу для трех машин - R744/744, R744/R717, $R 744 / R 290$, и задачи «транспортную» и «унификации» для машины $R 744 / 744$. Входные данные: холодопроизводительность $Q_{0}=100$ кВт, минимальная температура в газовом охладителе $T_{\min }^{\mathrm{BK}}=30^{\circ} \mathrm{C}$, температура кипения $T_{0}^{\mathrm{H \kappa}}=-50^{\circ} \mathrm{C}$, индикаторный КПД компрессоров $\eta_{i}=0,75$, давления в охладителе $\mathrm{p}_{\text {охл }}^{\text {вк }}=80 \ldots 100$ бар. Выбор входных данных произведен на основании анализа технической информации для одноступенчатых циклов с $R 744$ [8].

Итоги расчетов приведены в графической форме на рисунках 3 и 4.

Для машины $R 744 / 744$ (рисунок 3) степень термодинамического совершенства цикла при высоких температурах в конденсаторе-испарителе $T_{\kappa u}=-10 \ldots+2{ }^{\circ} \mathrm{C}$ имеет экстремальные значения, причем более высокие соответствуют меньшим давлениям в охладителе.

Массогабаритные характеристики машины не зависят от давления в газовом охладителе, а их минимальные значения наблюдаются при температу$\operatorname{pax} T_{\kappa и}=-15 \ldots-5^{\circ} \mathrm{C}$.

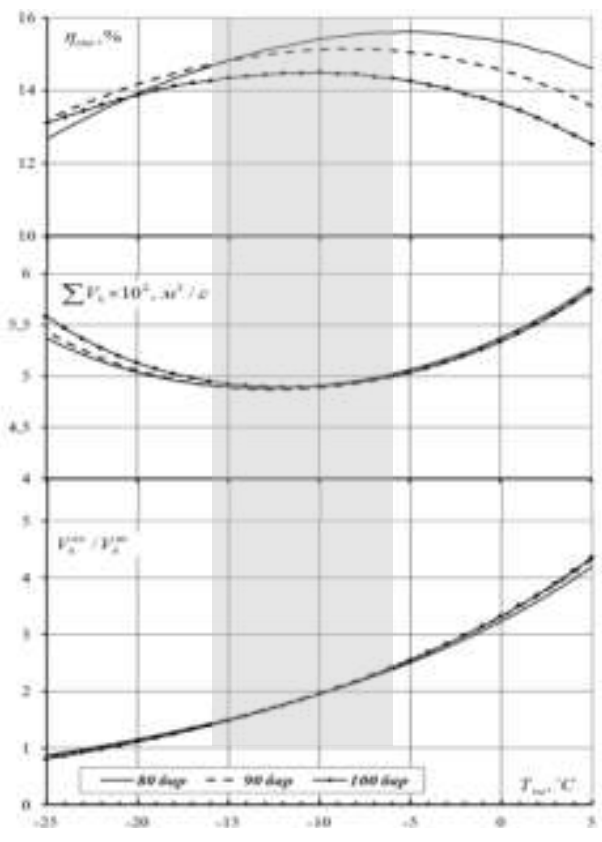

Рисунок 3 - Объемные и энергетические характеристики машины $R 744 / 744$ в зависимости от $p_{o x л}^{6 \kappa}$ и $T_{\kappa и}$
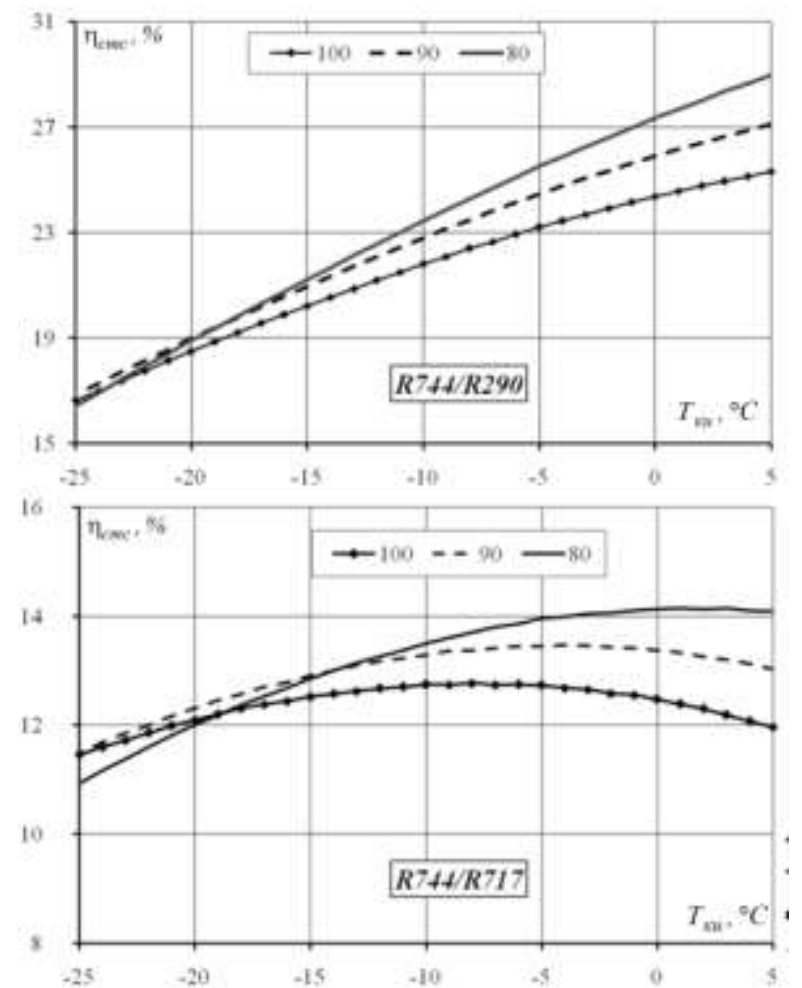

Рисунок 4 - Энергетические характеристики машин

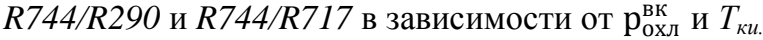

Обратим внимание на рассматриваемый интервал температур. При проектировании машины можно одновременно решить две задачи: «энергетическую» и «транспортную», если в газовом охладителе обеспечить давление $\mathrm{p}_{\text {охл }}^{\text {вк }}=100$ бар. При этом решением задачи «унификации» можно считать отношение 
теоретических объемных производительностей,

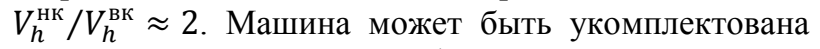
компрессорами одного унифицированного ряда, например, двух- и четырехцилиндровыми.

Обратимся к машинам $R 744 / R 717$ и $R 744 / R 290$ (рисунок 4).

Степень термодинамического совершенства в обеих машинах также зависит от давления в газовом охладителе. Более высокие значения относятся к низким давлениям в газовом охладителе при высоких температурах в кондесаторе-испарителе. При понижении температуры $T_{\kappa и}$ происходит изменение характера зависимости: в машине $R 744 / R 290 \eta_{\text {стс }}$ не зави-

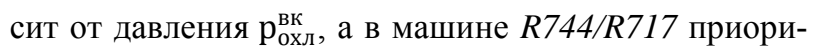
тет принадлежит высоким давлениям. В этой же машине наблюдаются экстремальные значения $\eta_{\mathrm{cmc}}$, аналогичные машине $R 744 / 744$.

Наивысшей степенью термодинамического совершенства обладает машина $R 744 / R 290$ со значениями на $25 . .40 \%$ выше остальных. На величину $\eta_{c m c}$ оказывают влияние положительные свойства $R 290$ как рабочего вещества холодильных машин.

\section{ВЫВОДЫ}

Получил дальнейшее развитие метод термодинамического анализа, который базируется на оценке степени термодинамического совершенства пары рабочих веществ, что позволяет осуществлять синтез схемно-цикловых решений каскадных холодильных машин, отвечающих задачам энергосбережения.

На примере машины $R 744 / 744$ разработан способ построения соответственных циклов-образцов для каскадных машин, в которых верхний каскад работает по надкритическому циклу.

Автором доказана применимость способа выбора $R 744$ в качестве рабочего вещества для каскадной машины, основанного на термодинамическом анализе объемных и энергетических характеристик машины, что дало возможность учитывать конкретное влияние свойств $R 744$ в каскадах на совершенство машины и достаточно точно определять оптимальные режимы ее работы.

Таким образом, на стадии предварительного проектирования без сложных вариантных расчетов обеспечивается рациональный выбор схемы и цикла для каскадной машины с $R 744$, соответствующий решению любой задачи: «энергетической», « транспортной» или «унификации».

\section{ЛИТЕРАТУРА}

1. Dopazo, A. Experimental Evaluation of a Cascade Refrigeration System Prototype with $\mathrm{CO} 2$ and $\mathrm{NH} 3$ for Freezing Process Application [Text] / A. Dopazo, J. Fe-
mandez-Seara // International Journal of Refrigeration. 2011. - Vol. 34, Issue 1. - P. 257-267.

doi: 10.1016/j.ijrefrig.2010.07.010

2. Bingming, W. Experimental Investigation on the Performance of NH3/C02 Cascade Refrigeration System with Twin-Screw Compressor [Text] / W. Bingming, W. Huagen, L. Jianfeng, X. Ziwen // International Journal of Refrigeration. - 2009. - Vol. 32, Issue 6. - P. 13581365.

doi:10.1016/j.ijrefrig.2009.03.008

3. Lee, T. Thermodynamic analysis of optimal condensing temperature of cascade-condenser in $\mathrm{CO} 2 / \mathrm{NH} 3$ cascade refrigeration systems [Text]/ T. Lee, C. Liu, T. Chen // International Journal of Refrigeration. - 2006. Vol. 29, Issue 7. - P. 1100-1108. doi: 10.1016/j.ijrefrig.2006.03.003 .

4. BitzerKühlmas chinenbau GmbH. [Электронный ресурс] / Обзор хладагентов. - 2004 - № 13.А501-13. - С. $36 . \quad$ - Режим доступа: http://ykaxolod.com.ua/file/Обзор\%20хладагентов\%20 и\%20их\%20взаимозаменяемость.pdf.

5. Bhattacharyya, S. Optimization of a $\mathrm{CO} 2-\mathrm{C} 3 \mathrm{H} 8$ Cascade System for Refrigeration and Heating [Text] / S. Bhattacharyya, A. Kumar, R. K. Khurana, J. Sarkat // International Journal of Refrigeration. - 2005. - Vol. 28, Issue 8. - P. 1284-1292.

doi: 10.1016/j.ijrefrig.2005.08.010

6. Di Nicola, G. Blends of Carbon Dioxide and HFCs as Working Fluids for the Low-Temperature Circuit in Cascade Refrigerating Systems [Text] / G. Di Nicola, G. Giuliania, F. Polonaraa, R. Stryjekb // International Journal of Refrigeration. - 2005. - Vol. 28, Issue 2. - P. 130-140. doi: 10.1016/j.ijrefrig.2004.06.014

7. Yamaguchi, H. Investigation of Dry Ice Blockage in an Ultra-Low Temperature Cascade Refrigeration System Using $\mathrm{CO} 2$ as a Working Fluid [Text] / H. Yamaguchi, X.-D. Niu, K. Sekimoto, P. Neksa // International Journal of Refrigeration. - 2011. - Vol. 34, Issue 2. - P. 466-475.

doi: 10.1016/j.ijrefrig.2010.11.001

8. Морозюк, Т. В. Теория холодильных машин и тепловых насосов [Текст] / Т. В. Морозюк. - Одесса: Студия «Негоциант», 2006. - 712 с.

9. Морозюк, Л.И. Оценка термодинамического совершенства рабочих веществ каскадных холодильных машин [Текст]/ Л.И. Морозюк // Восточноевропейский журнал передовых технологий. - 2015. 2/8 (74), c. 47-52.

doi: 10.15587/1729-4061.2015.39201

10. Мартыновский, В. С. Анализ действительных термодинамических циклов [Текст] / В. С. Мартыновский. - М.: Энергия, 1972. - 216 с.

11. Гайдук С. В. Розроблення та аналіз схемних рішень енергоперетворювальних систем, що працюють 3 діоксидом вуглецю [Текст]: дис. канд. техн. наук / С.В. Гайдук. - Одесса, 2014. - 176 с.

Отримана в редакції 09.10.2015, прийнята до друку 18.12.2015 
L. I. Morosuk M $^{\bowtie}$

Odessa National Academy of Food Technologies, 112 Kanatnaya str., Odessa, 65039, Ukraine

$\bowtie$ e-mail: lara.morozyuk@mail.ru, https://orcid.org/0000-0003-4133-1984

\section{CASCADE REFRIGERATION MACHINES WITH R744 AS THE WORKING FLUID FOR THE HIGH-TEMPERATURE CASCADE}

The cascade refrigeration machines are used for producing the cold at temperature level $-110 \ldots-50^{\circ} \mathrm{C}$. The thermodynamic cycle of the cascade refrigeration machine consists of one-stage refrigeration cycles in series. In earlier time, R12 or R22 were used as the working fluid for the high-temperature cascade as well as R13 or R14 as the working fluid for the low-temperature cascade. In modern refrigeration engineering these working fluids are forbidden. This fact requires to select another working fluids taking into account energy saving and ecological issues. R744, R717 and R290 (different combinations between high-temperature and low-temperature cascades) are the working fluids that can be used for generating the cold at temperature level $-30 \ldots-60^{\circ} \mathrm{C}$. They have the following advantages: the ODP values are equal to zero as well as GWP values tend to zero. In the paper, the thermodynamic analysis of the cascade refrigeration machines with $R 744 / R 744, R 744 / R 717, R 744 / R 290$ has been conducted with $R 744$ transcritical cycle for the high-temperature cascade. The thermodynamic analysis is based on the method of the comparison of the thermodynamic cycles proposed by R. Plank. For each cascade machine the operation parameters have been optimized to solve the "energetic problem". For the R744/R744 cascade refrigeration machine the "transport" and the "unification" problems are also solved. The parametric study of effect of the temperature within the condenser-evaporator on effectiveness of the thermodynamic cycles has been reported. The influence of the thermodynamic properties of the working fluids to the energetic and volumetric characteristics of the cascade refrigeration machines has also been evaluated.

Key words: cascade refrigeration machine $-R 744$ - transcritical cycle-energetic and volumetric characteristics

\section{REFERENCES}

1. Dopazo, A. 2011. Experimental Evaluation of a Cascade Refrigeration System Prototype with $\mathrm{CO} 2$ and NH3 for Freezing Process Application. International Journal of Refrigeration, 34, 257-267.

doi: 10.1016/j.ijrefrig.2010.07.010

2. Bingming, W. 2009. Experimental Investigation on the Performance of $\mathrm{NH} 3 / \mathrm{C} 02$ Cascade Refrigeration System with Twin-Screw Compressor. International Journal of Refrigeration, 32, 1358-1365.

doi:10.1016/j.ijrefrig.2009.03.008

3. Lee, T. 2006. Thermodynamic analysis of optimal condensing temperature of cascade-condenser in CO2/NH3 cascade refrigeration systems, 29, 1100-1108. doi: 10.1016/j.ijrefrig.2006.03.003 .

4. Bitzer Kühlmas chinenbau GmbH. Obzor hladagentov 2004, No.13. A-501-13. 36. Available at: http://ykaxolod.com.ua/file/Obzor\%20hladagentov\% 20-i\%20ix\%20vzaimozamenjaemost.pdf. Date of access: 17.10 .2016

5. Bhattacharyya, S. 2005.Optimization of a CO2C3H8 Cascade System for Refrigeration and Heating. International Journal of Refrigeration, 28, 1284-1292. doi: 10.1016/j.ijrefrig.2005.08.010
6. Di Nicola, G. 2005. Blends of Carbon Dioxide and HFCs as Working Fluids for the Low-Temperature Circuit in Cascade Refrigerating Systems. International Journal of Refrigeration, 28, 130-140.

doi: 10.1016/j.ijrefrig.2004.06.014

7. Yamaguchi, H. 2011. Investigation of Dry Ice Blockage in an Ultra-Low Temperature Cascade Refrigeration System Using $\mathrm{CO} 2$ as a Working Fluid. International Journal of Refrigeration, 34, 466-475.

doi: 10.1016/j.ijrefrig.2010.11.001

8. Morozjuk, T. V. 2006. Teorija holodil'nyh mashin i teplovyh nasosov, Studija «Negociant», 712.

9. Morozjuk, L. I. 2015. Assessment of thermodynamic perfection of working substancesin cascade refrigerators. Eastern-european journal of enterprice technologies, 2, 8(74), 47-52.

10. Martynovskij, V.S. 1972. Analiz dejstvitel'nyh termodinamicheskih ciklov, Energija, 216.

11. Haiduk, S. V. 2014. Rozroblennia ta analiz skhemnykh rishen enerhoperetvoriuvalnykh system, shcho pratsiuiut' z dioksydom vuhletsiu, Dys. kand. tekhn. nauk, $176 \mathrm{p}$. 